\title{
VISUAL RESPONSES OF SINGLE NEURONS IN THE CAUDAL LATERAL PULVINAR OF THE MACAQUE MONKEY ${ }^{1}$
}

\author{
LOUIS A. BENEVENTO ${ }^{2}$ AND JOHN MILLER \\ College of Medicine, University of Illinois at the Medical Center, Chicago, Illinois 60680
}

\begin{abstract}
Single unit recordings were made in the portion of the lateral pulvinar which forms the lateral aspect of the caudal pole of the thalamus, i.e., $\mathrm{PL}_{\gamma}$ (Rezak, M., and L. A. Benevento (1977) Soc. Neurosci. Abstr. 3: 573; Rezak, M. (1978) Soc. Neurosci. Abstr. 4: 642), of macaque monkeys. PL $_{\gamma}$ receives convergent inputs from the occipital cortex and has strong reciprocal interconnections with the visual association cortex, including the inferotemporal cortex (areas 20 and 21). It was found that $\mathrm{PL}_{\gamma}$ has a poor or nonexistent retinotopic organization. Many of the neurons had large, unflanked, overlapping receptive fields which often included the fovea. A few neurons could be influenced by a visual stimulus placed anywhere in the visual field described by a tangent screen. The receptive fields could be bilateral or located entirely within the contralateral or ipsilateral hemifields. The majority of units were binocular and exhibited various types of binocular interaction which could be quite complex. The binocular response was not predictable from the algebraic sum of the monocular responses and could be of the opposite sign (e.g., excitatory when the monocular response was inhihitory). Neurons which were also sensitive to the direction of movement of stimuli projected upon the tangent screen formed a major group. Of the units sensitive to tangentially moving stimuli, two special subgroups were found. One group of neurons gave sustained responses to static levels of luminance, while the other group was sensitive to stimuli which moved toward or away from the eyes. The nonlinear rate of change of the apparent size of approaching or receding stimuli was described by a mathematical function which also describes the response of the neurons to the same stimuli. For many of these units which were sensitive to tangentially moving stimuli and one other class of stimuli, such as luminance levels or movement in depth, the responses to one class were seemingly unrelated to the responses to the other class. The same statement may be made for monocular and binocular responses. It may be, then, that different wiring diagrams describe these different types of inputs. These physiological results are discussed in terms of the inputs to $\mathrm{PL}_{\gamma}$ as well as its cortical targets.
\end{abstract}

In simians, the pulvinar complex is usually divided into lateral, medial, and inferior subdivisions (Walker, 1938). In recent years, the connections of these regions in Old World monkeys have become better known, and these regions have been subdivided further and defined based on their individual patterns of inputs and outputs (e.g., Rezak and Benevento, 1977, 1979). However, to date, only a few studies have investigated the physiological properties of the pulvinar in Old World monkeys, and, as yet, nothing is known about the functional properties of

\footnotetext{
'We wish to thank Mrs. Bhavna Shah for her technical assistance and Drs. Robert Jaeger and Sidney Yates for their help with the mathematics. We are also grateful to Drs. Gary Felsten and Gregg Standage for their comments on the manuscript. This work was supported by National Institutes of Health Grant EY 2940. John Miller was supported by a University of Illinois assistantship.

${ }^{2}$ To whom correspondence should be addressed at Department of Anatomy, College of Medicine, University of Illinois at the Medical Center, P.O. Box 6998, Chicago, IL 60680.
}

pulvinar cells in relation to their anatomical connections. The lateral pulvinar, in particular, has more than one subdivision, each of which is organized differently (Rezak and Benevento, 1977, 1979). These regions of the lateral pulvinar, in turn, provide inputs to various areas with visual and visuomotor properties which are located in the occipital, temporal, and parietal lobes (Benevento and Rezak, 1976; Benevento et al., 1977a; Rezak and Benevento, 1979; Standage and Benevento, 1981).

In rostral regions of the pulvinar complex, both the inferior pulvinar and the adjacent portion of the lateral pulvinar (known as region $\mathrm{PL}_{\alpha}$; Rezak and Benevento, 1977) contain representations of the contralateral visual hemifield, have topographical interconnections with the visual cortex (Benevento and Rezak, 1976; Benevento and Davis, 1977; Rezak and Benevento, 1979), and contain neurons with visual response properties and discrete receptive fields (Felsten et al., 1981). Outside of this rostral region, the organization is different. Caudally, the 
inferior pulvinar is absent and the organization of the lateral pulvinar (which now forms the lateral division of the caudal pole of the thalamus) changes. This portion of the lateral pulvinar, which has been called $\mathrm{PL}_{\gamma}$ (Rezak and Benevento, 1977; Rezak, 1978), receives convergent input from wide regions of visual cortex (Benevento and Davis, 1977) and has heavy projections to the visual association cortex, such as the inferotemporal cortex (Rezak, 1978; Benevento and Rezak, 1976). The inferotemporal cortex is very important for attention and discrimination (e.g., Chow, 1954; Ettinger et al., 1968; Iwai and Mishkin, 1969) but has been shown to have a very poor retinotopic organization (Desimone and Gross, 1976), which is probably a result of convergent cortical and subcortical inputs. Of the subcortical regions which provide input to the inferotemporal cortex, it is the caudal lateral pulvinar, or $\mathrm{PL}_{\gamma}$, which acts as the link in corticothalamocortical connections between this area and other visual association cortices.

'The present report focuses on this caudal aspect of the lateral pulvinar since, to our knowledge, there are no reports on the response properties of the neurons there and, as just stated, this region represents a major link in the corticothalamocortical interconnections of the visual association cortices. The results of the present study illustrate properties of neurons which are not typically reported in the mammalian visual system. They include large, bilateral receptive field organizations, sensitivity to changes in luminosity levels and to various types of movement (including three dimensional), and complex binocular interactions. One interesting aspect of this study is the finding that, for many of these units, the responses to one class of stimuli is apparently unrelated to the responses to another class, implying that these neurons receive multiple convergent, but functionally distinct, afferent inputs.

\section{Materials and Methods}

Animal preparation. Five Macaca fascicularis weighing 2.5 to $3.5 \mathrm{~kg}$ were used simultaneously for the present study and for neuroanatomical studies (reported elsewhere) which involved injections of horseradish peroxidase or tritiated amino acids in regions of the brain other than the pulvinar.

Our recording procedure for acute preparations has been reported in detail before (e.g., Benevento et al., 1972; Benevento et al., 1977a; Fallon and Benevento, 1977; Fallon et al., 1978). The animals were anesthetized for surgery initially with halothane and stereotaxic pressure points and incision margins were infiltrated with lidocaine. For the duration of the experiment, local anesthesia was maintained by periodic infiltrations of lidocaine and general anesthesia was preserved by artificial respiration with a $30 \% \mathrm{O}_{2}, 70 \% \mathrm{~N}_{2} \mathrm{O}$ mixture. The EEG and EKG also were monitored continuously to note the level of anesthesia and condition of animal. As further precautions against pain and to free the ears and eyes, the stereotaxic ear, eye, and mouth bars were removed and the head was held by modified semichronic stereotaxic head tubes (Kopf Instruments, No. 880) which were cemented to the skull. A stainless steel recording chamber was fitted into a hole in the skull and fixed in place with dental cement. The chamber was filled with warm $\left(37.5^{\circ} \mathrm{C}\right)$ Ringer's solution, and a threaded cap with a plastic insert was used to seal the chamber. Reflection of the dura and hydraulic sealing of the recording chamber were done just prior to the recording session. Rectal temperature was monitored and maintained at 37.5 to $38.5^{\circ} \mathrm{C}$. During the recording sessions, the animals were immobilized by continuous intravenous injection of a mixture of gallamine triethiodide and tubocurarine in $5 \%$ glucose/Ringer's solution. Extracellular recordings were made with varnish-coated tungsten electrodes (2- to 8$\mu \mathrm{m}$ tips).

The electrodes were introduced into a small hole in the plastic insert of the recording chamber and later advanced into the brain with the aid of a hydraulic stepping microdrive (Kopf Instruments). The recorded action potentials were amplified and displayed on dual beam oscilloscopes (Tektronix), monitored on an audiomonitor (Grass Am8), and recorded on magnetic tape (HewlettPackard). On-line and off-line analyses in the form of post-stimulus time histograms (PSTH) were made with a PDP-8/E computer. At several points during each penetration, small electrolytic lesions were made to assist with the identification and reconstruction of electrode tracts. In addition, the locations of units of particular interest also were marked by lesions. The lesions were used as reference points from which distances along the penetration were measured to reconstruct the location of each unit.

For visual stimulation, the animal's pupils were dilated with an ophthalmic solution of Neo-Synephrine (phenylephrine) and Mydriacyl (tropicamide). The eyes were refracted with a refractometer (Rodenstock) to select the contact lenses needed to correct vision for a $0.5-$ to $1-\mathrm{m}$ distance. Prisms were used to align the visual axes of the eyes to obtain correspondence of the visual fields at this distance. The positions of the fovea and optic discs were plotted on translucent white paper attached to a tangent Plexiglas screen at a distance of 0.5 to $1 \mathrm{~m}$ by means of back projection via an ophthalmoscope. The position of the midpoint of the fovea in the visual field was checked systematically and used as a reference point for determining the eccentricity of the visual receptive fields.

Monocular and binocular visual stimuli were displayed on the tangent screen by projectors. These "automated" stimuli were created by projecting images onto a first surface mirror which was attached to a galvanometer. Movement of the stimuli across the screen was accomplished by linearly driving the galvanometer with the output of a W-P Instruments function generator which also was synchronized with the trigger of a PDP/E computer. For flashes, the onset and offset of stimuli were accomplished by means of an electronic shutter which was controlled by the same function generator coupled to the computer. The stimuli included large field or small field flashes of white light, oscillating grids and light or dark visual targets of various shapes, orientations, and directions of movement. These stimuli were usually 0.5 to $1.5 \mathrm{log}$ units above the intensity of mesopic background illumination $\left(10^{-1} \mathrm{~cd} / \mathrm{m}^{2}\right)$. Light intensity was attenuated with neutral density filters.

The receptive field boundaries were determined by 
plotting the location of the initial minimum response shown on the PSTH for each of the stimulus trajectories which were spaced at approximately $45^{\circ}$ intervals.

For testing responses of units to approaching or receding stimuli, various two-dimensional shapes were mounted on an X-Y plotter driven by a sawtooth from a waveform generator. In this way, the shapes were driven linearly toward or away from the animal while being silhouetted by background illumination.

A number of units also were tested for sensitivity to auditory and somatosensory stimulation. The main emphasis of the present study was on visual responses so that auditory and somatosensory stimuli were given in a relatively cursory fashion. Dichotic tone and click stimuli were administered through car phones embedded in the ears as described in detail before (e.g., Benevento and Coleman, 1970; Fallon et al., 1978). Somatosensory stimulation was administered by brushing the animal's body.

After the recording sessions, the animals were anesthetized deeply with pentobarbital sodium and perfused transcardially with $10 \%$ formalin or buffered $4 \%$ glutaraldehyde/paraformaldehyde. The brains were blocked in the stereotaxic transverse planes, frozen-sectioned at 30 $\mu \mathrm{m}$, and stained with toluidine blue. The sections were studied under the microscope for reconstruction of electrode tracks (Fig. 1).

\section{Results}

The present results were taken from 236 isolated single unit recordings located in the caudal third of the lateral pulvinar $\left(\mathrm{PL}_{\gamma}\right) . \mathrm{PL}_{\gamma}$ extends from the level of the superior colliculus to the caudal end of the inferior pulvinar (Fig. 1) and forms the lateral aspect of the caudal pole of the thalamus. Sixty-eight percent of the units encountered throughout the dorsoventral extent of the lateral pulvinar, at these levels, were influenced by visual stimuli.

Visually responsive neurons were characterized by means of both moving hars of light and stationary flashes. A great majority of the visual units $(88.5 \%)$ gave strong responses to moving stimuli (referred to as "movement sensitive"). Stimulation of the receptive fields of these movement-sensitive units with stationary flashing stimuli would produce a phasic or sometimes tonic response which could, in some cases, be as strong as the response to moving stimuli. A few of these neurons seemed to be insensitive to flashing stimuli. Another group of visually responsive neurons $(11.5 \%)$ gave a strong sustained response to illumination of the entire visual field (or large portions of it) and a comparatively weaker response to moving stimuli.

Receptive fields. The units had large receptive fields ranging from about $10^{\circ}$ to $60^{\circ}$ in diameter. The limits of the receptive fields were plotted with automated stimuli as described under "Materials and Methods." Because the response of the movement-sensitive cells to stationary flashing stimuli was particularly labile in our preparations, it is difficult to compare the effects of stationary and dynamic stimuli with the present data (but see Felsten et al., 1981). It should be noted that recent experiments which used chronic, instead of acute, preparations indicate that a phasic or tonic response to flashing stimuli is a frequently found characteristic of lateral pulvinar units in macaque monkeys (Felsten et al., 1981). The results from the use of both automated and handdelivered stimuli indicated that no clear surrounds or flanks of the opposite sign (e.g., excitation versus inhibition) were obvious within the receptive fields. Stationary, discrete flashes revealed that the receptive fields of a few units had an inner area, displaced from the center, which gave a more intense but similar (i.e., of the same sign) response than the remainder of the receptive field. For some units, the limits of the receptive field could not be determined, but the firing pattern could be influenced by moving a light stimulus over most of the tangent screen.

Since it was frequently not possible to locate receptive field boundaries precisely by means of hand-delivered stimuli, it was difficult to position the automated stimuli so that they were centered on the receptive fields (e.g., Fig. 3). This could raise the charge, then, that the responses to different directions of movement and orientations of the stimulus may be distorted due to failure of the stimulus sweeps to cover all portions of the receptive field. This danger can be discounted for several reasons. The positions of the initial minimal responses on the PSTHs usually could be plotted in a systematic manner, and the receptive field geometry so derived did not reveal any gaps or irregularities. Moreover, the responsive regions on the PSTHs (inhibition or excitation) were well contained between the beginning and end of each stimulus sweep. If a portion of a receptive field was missed by a stimulus sweep along one trajectory, due to inadequate stimulus length, this fact was revealed by presenting the same stimulus along a second, perpendicular trajectory. Thus, the true length of the receptive field in the direction orthogonal to the first trajectory would be disclosed. If any irregularities were detected, the stimulus bars were lengthened or repositioned accordingly. Since lengthening the stimuli beyond receptive field boundaries did not appear to affect the responses of our units, we generally used very long bars or slits to avoid missing portions of the receptive fields.

Visuotopic organization. Although the retinotopic organization of the rostral portion of the lateral pulvinar which surrounds the inferior pulvinar (region $\mathrm{PL}_{\kappa}$; Rezak and Benevento, 1977) is clear (Fig. 1; e.g., Benevento and Davis, 1977; Rezak and Benevento, 1979), we found that the caudal portion studied here, $\mathbf{P L}_{\nu}$, had, in contrast, a disorganized and poor representation of the visual field and, additionally, a number of units with bilateral receptive fields which could encompass the fovea (Fig. 1; Table I).

The penetrations diagrammed in Figure 1 are meant to illustrate the location of the units whose responses are illustrated in Figures 3 through 6 . The types and locations of the receptive fields shown in Figure 1, however, are representative of the organization seen in $\mathrm{PL}_{\gamma}$. About half of our data were useful for reconstruction of the penetrations and what was seen consistently, as one proceeds ventrally in $\mathrm{PL}_{\gamma}$, was that the receptive fields jump in position from peripheral to central and vice versa (Fig. $1, B$ and $C$ ).

Many of the receptive fields overlapped. The centers of the bilateral receptive fields were usually in the contralateral hemifield, but for some neurons, the center 
TABLE I

Characteristics of visually responsive units

Categories I, II, and III refer to $80 \%$ of the 160 visually responsive units which had definable receptive field boundaries. The numbers in categories IV, V, VI, VII, and VIII are expressed as percentages of the total of 160 visually responsive units.

\begin{tabular}{clc}
\hline Category & \multicolumn{1}{c}{ Characteristic } & Measurement \\
\hline I & Mean receptive field diameter & $24.7^{\circ}$ \\
II & $\begin{array}{l}\text { Receptive fields located in area of } \\
\text { central vision }\end{array}$ & $45.2 \%$ \\
III & Bilateral receptive fields & \\
& Receptive fields in contralateral & $47.1 \%$ \\
& $\quad$ hemifield & $47.1 \%$ \\
& Receptive fields in ipsilateral & $5.8 \%$ \\
& hemifield & \\
IV & Unidirectional & $60.7 \%$ \\
& Nondirectional & $39.3 \%$ \\
V & Optimal rate per sec & \\
& $\quad$ Fast (150 $-200^{\circ}$ ) & $50.0 \%$ \\
& Slow ( $\left.5^{\circ}-80^{\circ}\right)$ & $41.6 \%$ \\
& Over $300^{\circ}$ & $8.4 \%$ \\
VI & Binocular interaction evident & $83.3 \%$ \\
VII & Responds to motion toward or & $8.6 \%$ \\
& away & \\
VIII & Tonic response to luminance level & $11.5 \%$ \\
\hline
\end{tabular}

could be on the vertical meridian or well within the ipsilateral hemifield (Fig. 1). Receptive fields located entirely within the ipsilateral hemifield were found also.

Binocular interaction. Binocular interactions of various types were seen in the vast majority of units. The most common ocular dominance was from the contralateral eye. The histogram in Figure 2 summarizes the types of binocular interaction seen. Binocular inhibition and facilitation were both evident throughout $\mathrm{PL}_{\gamma}$. The binocular interaction could be quite complex and usually was not predictable from the algebraic summation of the monocular responses. Figure 3 illustrates one example of a complex binocular interaction in which a moving bar of light caused inhibition with the ipsilateral eye open and had little apparent effect with only the contralateral eye open but caused excitation with both eyes open. This unit was located ventrally in the caudal lateral pulvinar (Fig. 1).

Moving stimuli. The majority of units in $\mathrm{PL}_{\gamma}$ responded to tangentially moving stimuli and many were directionally selective (unidirectional); i.e., the responses for the two directions of movement along the same trajectory were asymmetrical (Table I). Another group of units $(39.3 \%)$ was not directionally selective; i.e., the responses for the two directions along the same trajectory were equivalent (Fig. 4).

On the other hand, for all visually responsive $\mathrm{PL}_{\gamma}$ neurons, excitation or suppression which resulted from the specific orientation of a stimulus was difficult to demonstrate and often difficult to dissociate from the response due to directional selectivity (Benevento et al.,
1972). Clearly, the majority of those units which were not directionally selective (or $33.4 \%$ of all visually responsive units) also did not display any orientation selectivity; i.e., for each stimulus orientation, the response was the same (Fig. 4A). Of the remaining visually responsive cells $(66.6 \%)$, there were no neurons whose responses were narrowly tuned to the orientation of a moving bar or line such as seen in the striate cortex, for example. Rather,
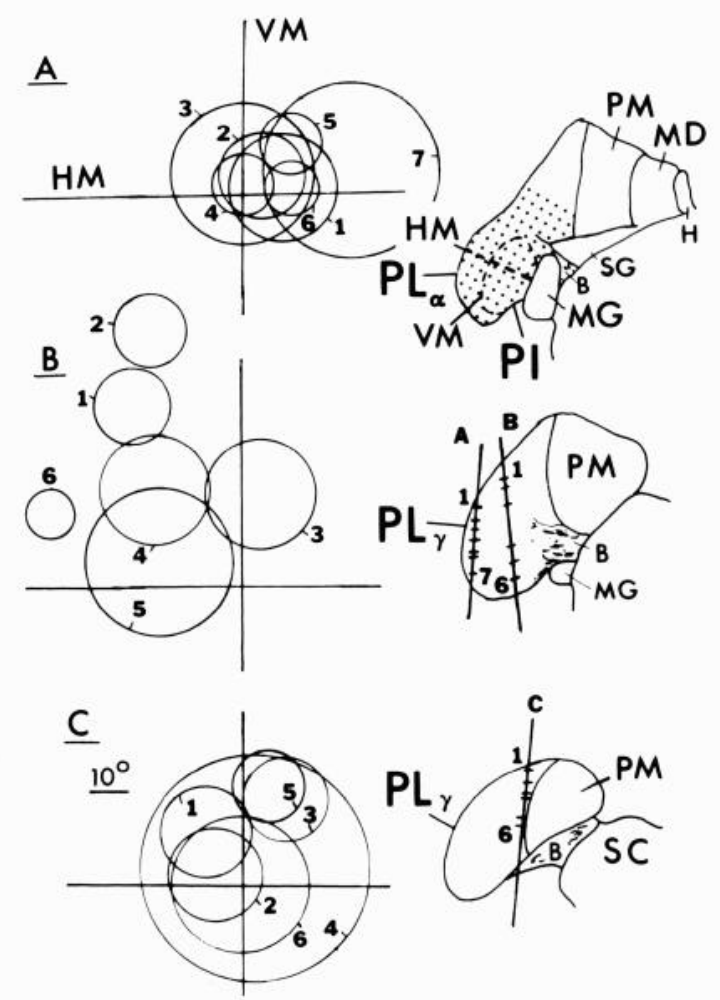

Figure 1. Receptive fields and location in $\mathrm{PL}_{\gamma}$. These drawings of transverse sections through the pulvinar complex of a macaque monkey are shown in order to illustrate the recording sites of the units whose responses are illustrated in Figures 3 to 6. Top is most rostral; bottom is most caudal. The lettering of each electrode tract $(A, B$, and $C)$ corresponds to the lettering of the receptive field plots on the left. Electrode tracts were reconstructed by the methods described in text. The most rostral level $(t o p)$ is shown for orientation. It is at this level that retinotopically organized regions in the lateral pulvinar $\left(P L_{\alpha}\right)$ and adjacent inferior pulvinar $(P I)$ exist (stippling). Note that the representation of the vertical meridian $(V M)$ marks the boundary between $P L_{\alpha}$ and $P I$ and that the horizontal meridian $(H M)$ bisects the two regions (from Benevento and Rezak, 1976; Benevento and Davis, 1977; Rezak and Benevento, 1977, 1979). Caudal to these regions is found $P L_{\gamma}$. The unit illustrated in Figure 3 is located in penetration $A$ at position $7(A 7)$. The unit in Figure 4 is located at $C 2$, the unit in Figure 5 is located at $B 5$, and the unit in Figure 6 is located at $C 4$. Some of the neurons with diffuse receptive fields, which could be as large as the tangent screen, are located next to levels $A 3, A 4$, and $C 3$. In the receptive field graphs, the contralateral hemifield is located to the left of the vertical meridian. Receptive fields are plotted as described in text. Other abbreviations used on the figure are: $B$, branchium of the superior colliculus; $H$, habenula; $M G$, medial geniculate nucleus; $M D$, dorsomedial nucleus; $P M$, medial pulvinar; $S C$, superior colliculus; $S G$, suprageniculate nucleus. 


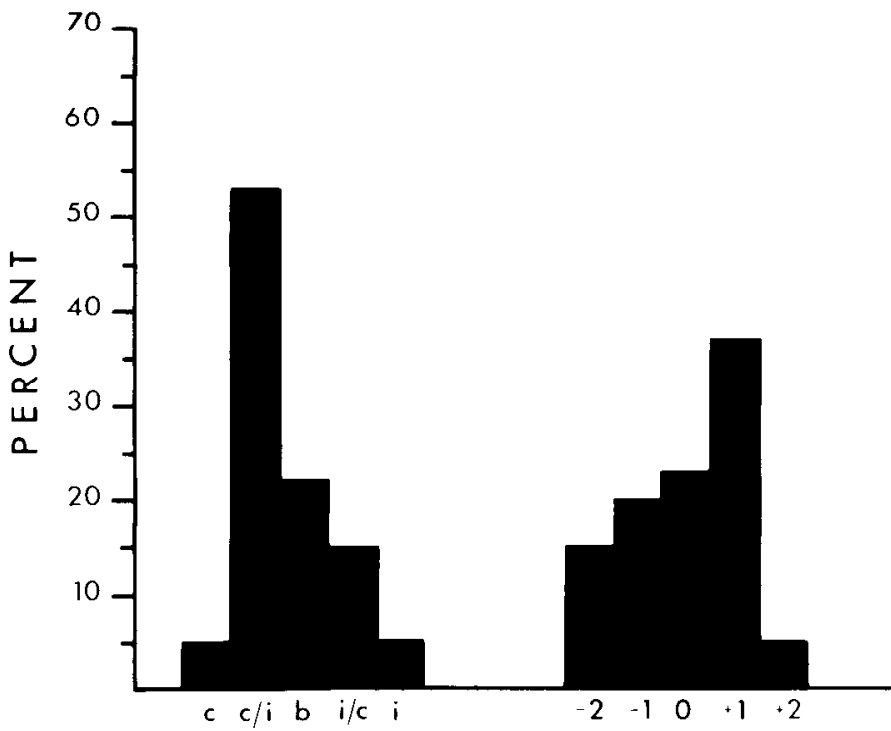

Figure 2. Bar graphs which summarize the types of binocular interaction encountered in single neurons located in $\mathrm{PL}_{\gamma}$. Ocular dominance is represented on the left and various types of binocular interaction are shown on the right. The ordinate represents the percentage of the 160 visual units studied. On the left, $c$ and $i$ represent the number of units which are driven by only the contralateral eye or only the ipsilateral eye, respectively. $b$ represents the number of units which are driven equally well by either eye; $c / i$ and $i / c$ represent the number of units which are driven more by the contralateral or ipsilateral eye, respectively. For the bar graph on the right, 0 represents the number of units in which the binocular response was equal to the algebraic sum of the monocular responses. For columns +1 and +2 , the response is greater than this sum, while for columns -1 and -2 , the response is less than this sum. For these categories, +2 and -2 represent a degree of binocular facilitation or inhibition apparently greater than +1 and -1 , respectively.

any sensitivity to the orientation of a stimulus was over a broad range $\left(45^{\circ}\right.$ to $\left.135^{\circ}\right)$ (e.g., Fig. $5 A$ ). Only a few of these units were not directionally selective. Pulvinar units with such broad ranges of orientation selectivity have been described in New World monkeys and have been called "non-oriented" by some authors (e.g., Gattas et al., 1979). Our data indicate that broad, poor orientation tuning is a prevalent finding in the caudal lateral pulvinar of the macaque.

Most movement-sensitive units which responded to tangentially moving stimuli could be broadly grouped into two different ranges of preferred velocity: 5 to $80^{\circ} \%$ sec and 150 to $200^{\circ} / \mathrm{sec}$. The neurons which had a preferred velocity in one of these groups also were somewhat sensitive to a wide range of velocities including the other velocity group. We also found some units whose velocity sensitivities could exceed $300^{\circ} / \mathrm{sec}$.

Special cases. In addition to the properties just described, we also found two other classes of units scattered throughout $\mathbf{P L}_{\gamma}$ which were somewhat unusual. One group was sensitive to changes in steady state luminance and the other was sensitive to a stimulus which moved toward or away from the animal (Table I).

Figure 4 illustrates a unit located in ventral $\mathrm{PL}_{\gamma}$ (Fig. 1) which is representative of a group of units that fired continuously to dark or light whole field illumination as well as to moving stimuli. This type of unit had large receptive fields (the smallest being $12^{\circ}$ in diameter) and was affected by either monocular or binocular luminance changes. In some cases, the borders of the receptive field were not definable and the unitary activity seemed influenced by large moving stimuli placed anywhere on the tangent screen. Furthermore, these units did not respond well to small stationary stimuli, even when they were confined to the receptive field (i.e., when the receptive field could be defined by automated moving stimuli). On the other hand, very large stationary stimuli could produce a response regardless of whether the receptive field (as defined by automated stimuli) was illuminated. Such responses were similar to, though weaker than, the response to whole field illumination. Thus, for these units, sensitivity to luminance level appeared to be a property of the whole visual field and not of any subdivision of the visual field. These units did not necessarily exhibit orientation or direction preferences, although very fast stimulus sweeps did alter the spike discharge. As with a number of pulvinar units encountered in this study, the receptive fields, or areas sensitive to changes in luminosity, included the fovea and about half of the units clearly had bilateral receptive fields.

Figure $4 A$ illustrates the response of such a unit to a moving bar of light. 'The excitatory response was similar for all directions and orientations. Panels $B$ and $C$ of Figure 4 illustrate the monocular responses of the unit during long periods of darkness and light. In contrast to the moving bar of light, the response was inhibited by whole field steady state illumination of the contralateral eye. The activity returned to resting levels during the dark. Steady state illumination or darkness did not seem to affect the unit when only the ipsilateral eye was open.

Figure 5 illustrates the responses of a different unit which exhibited an inhibitory response when presented with a bar of light which was moving in the proper direction. During constant illumination of the ipsilateral eye, however, the unit gave an increased excitatory response which returned to near spontaneous levels in the dark. Constant light or dark did not appear to affect the unit when only the contralateral eye was open. Thus, as seen with the examples given in Figures 4 and 5, it was not possible to predict the response to dynamic stimuli from the response to static stimuli and this may mean that there are different wiring diagrams for these two types of inputs.

Figure 6 illustrates another type of unit found in $\mathrm{PL}_{\gamma}$. This unit is representative of a type of unit which responded to tangentially moving bars of light, had directional selectivity and broad orientation tuning, and also responded to light or dark stimuli which moved toward or away from the eyes. The latter effect could be monocular or binocular. The receptive fields of these units ranged from $15^{\circ}$ to $55^{\circ}$ in diameter and some were also bilateral.

The units responded to a bright or dark spot which was expanded and contracted on the tangent screen in a way similar to approaching or receding stimuli. While these units also could respond to stationary flashing stimuli within the receptive field, the responses to stimuli 

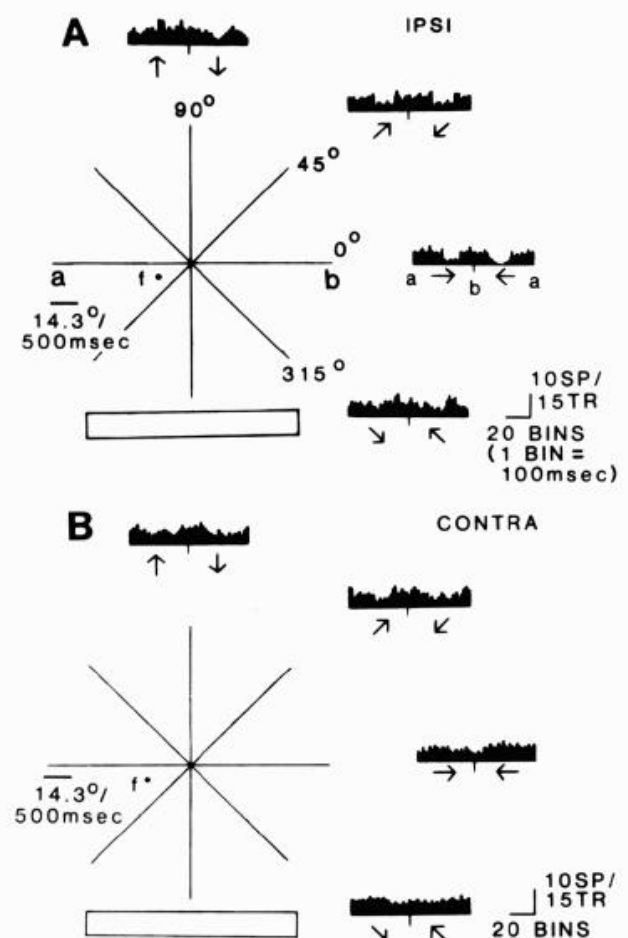

CONTRA
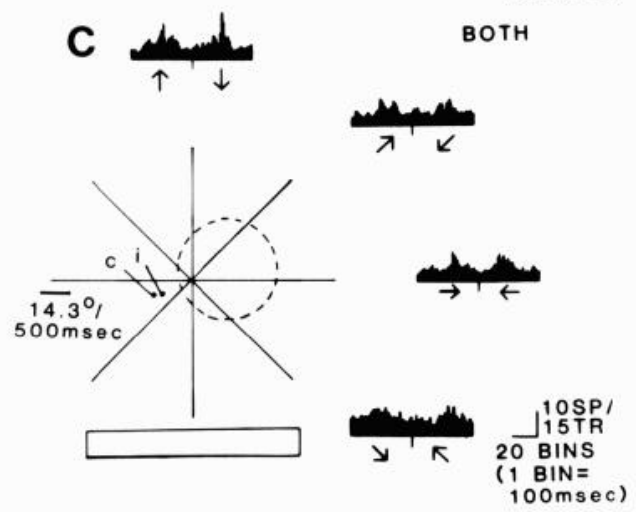

Figure 3. Binocular interaction. In this and the following figures, the intersecting lines, spaced at $45^{\circ}$ intervals, represent the trajectories for the visual stimulus shown at their bottom. The dashed line represents the position of the receptive field in relation to the stimulus trajectories $(C)$. Under each histogram, each of the two arrows denotes the stimulus direction which generated that half of the histogram (tick mark divides each PSTH in half). For each direction, the movement of the stimulus is over the entire distance indicated by the radial lines. For example in $A$, the left half of the histogram for the $0^{\circ}$ direction is generated when the line stimulus sweeps from point $a$ to point $b$. The right half of the histogram is generated when the stimulus returns from point $b$ to point $a$. Although the right half of all histograms represents the return direction of the stimulus, they are read from left to right. The stimulus bar is $10.7^{\circ} \times 129^{\circ}$ in size. Separate calibration bars label both the PSTHs and the stimulus trajectories upon which the receptive fields are plotted. On the left side of each plot of the stimulus trajectories, a single bar is used to calibrate distance in degrees of visual angle, time in milliseconds, and velocity. For example, the calibration bar in plot $A$ is $14.3^{\circ}$ in length, which is the distance traveled by the stimulus in the time given in the denominator $(500 \mathrm{msec})$. From this, the stimulus velocity, $14.3^{\circ} / 500 \mathrm{msec}$ or $28.6^{\circ} / \mathrm{sec}$, can be derived. The PSTH cali-

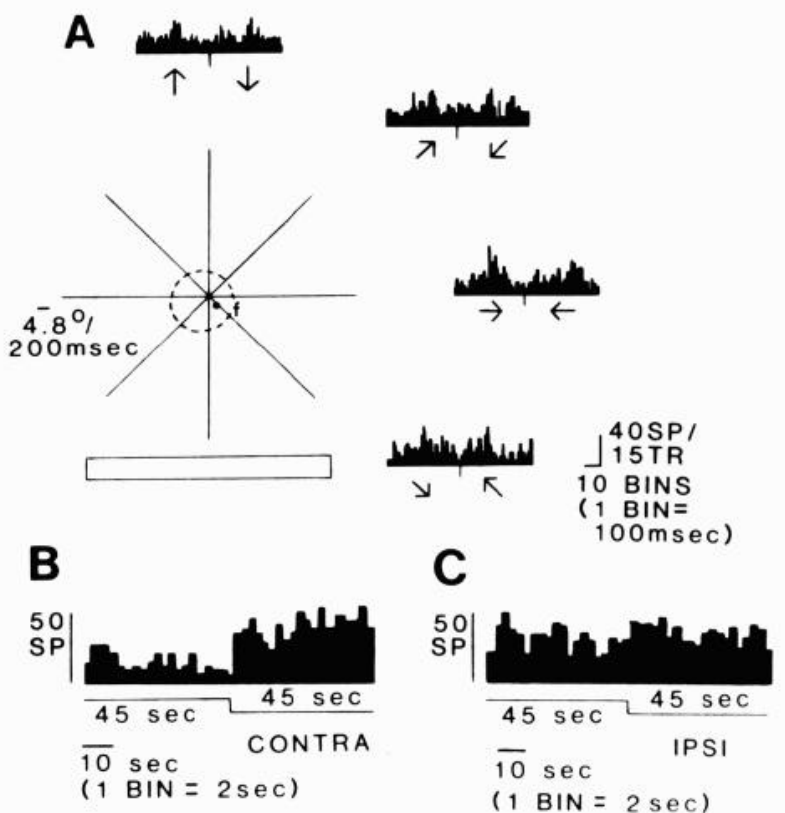

Figure 4. Sustained responses to steady illumination-inhibition. Response of a $\mathrm{PL}_{\gamma}$ unit to a moving bar of light $94.5^{\circ} \times$ $9.0^{\circ}$ in size $(A)$ and whole field illumination (45 sec on, $45 \mathrm{sec}$ off; $B$ and $C$ ). The bar in $A$ is moving at a velocity of $24^{\circ} / \mathrm{sec}$. Both monocular and binocular conditions gave a similar excitatory response to all directions of movement and orientations. The contralateral eye is shown in $A$ for comparison to the static conditions in $B$ where a $45-\mathrm{sec}$ steady illumination of the contralateral eye inhibits the units. The activity returns to "spontaneous levels" when the light is off. The ipsilateral eye seems unaffected by luminance changes. The response to luminance changes in the binocular condition is virtually identical to that of the contralateral conditions. Note that the bin width and ordinate are different in $A$ from those in $B$ and $C$. See Figure 1 for location of the unit.

moving toward or away from the animal and to expanding or contracting stimuli were much stronger. Thus, the ability of these units to respond to the changing apparent size of a stimulus is not simply a manifestation of changes in luminance.

Since these units do respond well to expanding and

brations are found on the lower right. The histograms represent the summation of the total number of trials $(T R)$ (in this case, 15). The vertical calibration bar represents an arbitrary number of spikes $(S P)$ (in this case, 10) which is appropriate for interpretation of the PSTHs. The horizontal bar calibrates the time base for each PSTH. The histograms are divided into bins measured in milliseconds (in this case, $100 \mathrm{msec}$ ) where 1 bin equals $100 \mathrm{msec}$. The size of the horizontal calibration bar in $A$ would equal $20100-$ msec bins or 2 sec. Note that, for $A$ (ipsilateral (IPSI) eye only), there is obvious inhibition with the $0^{\circ}$ and $45^{\circ}$ stimuli, while in $B$ (contralateral (CONTRA) eye only), inhibition of any sort is not obvious. In the binocular condition $(C)$, all stimuli give an obvious peak excitation. The receptive field for the binocular condition is the only one shown; i.e., the only one where the borders were obvious due to clear excitatory responses. See also Figure 1 for location of the unit. $c$ and $i$ indicate the positions of the foveas for the contra- and ipsilateral eyes during binocular stimulation; $f$ indicates the foveal position during monocular stimulation. 

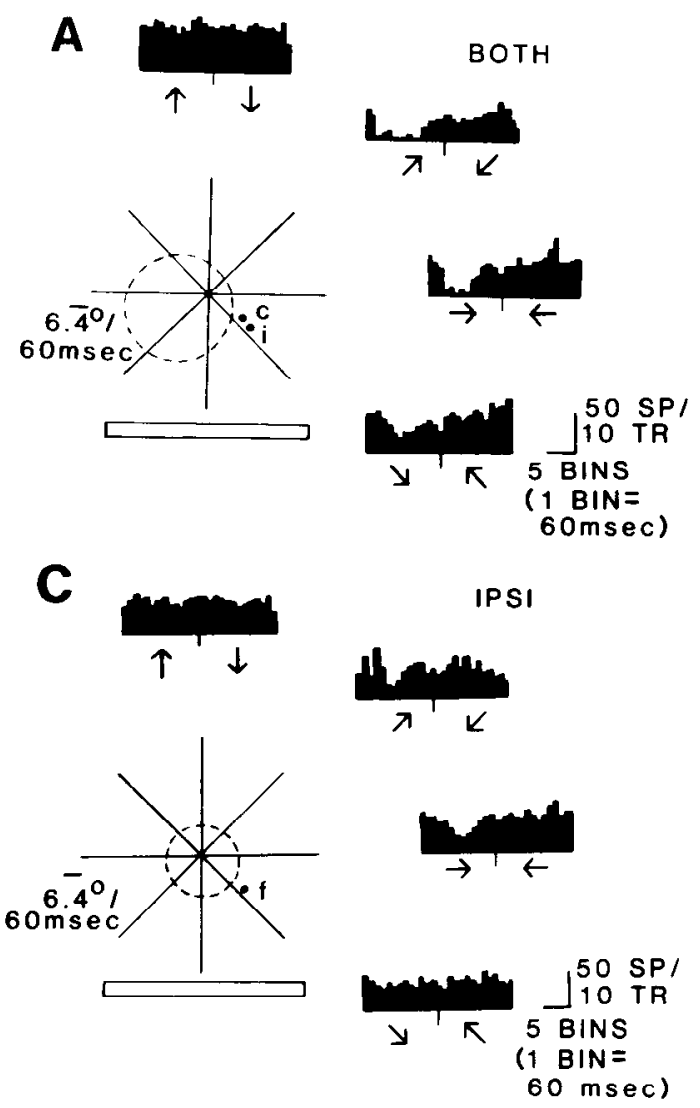
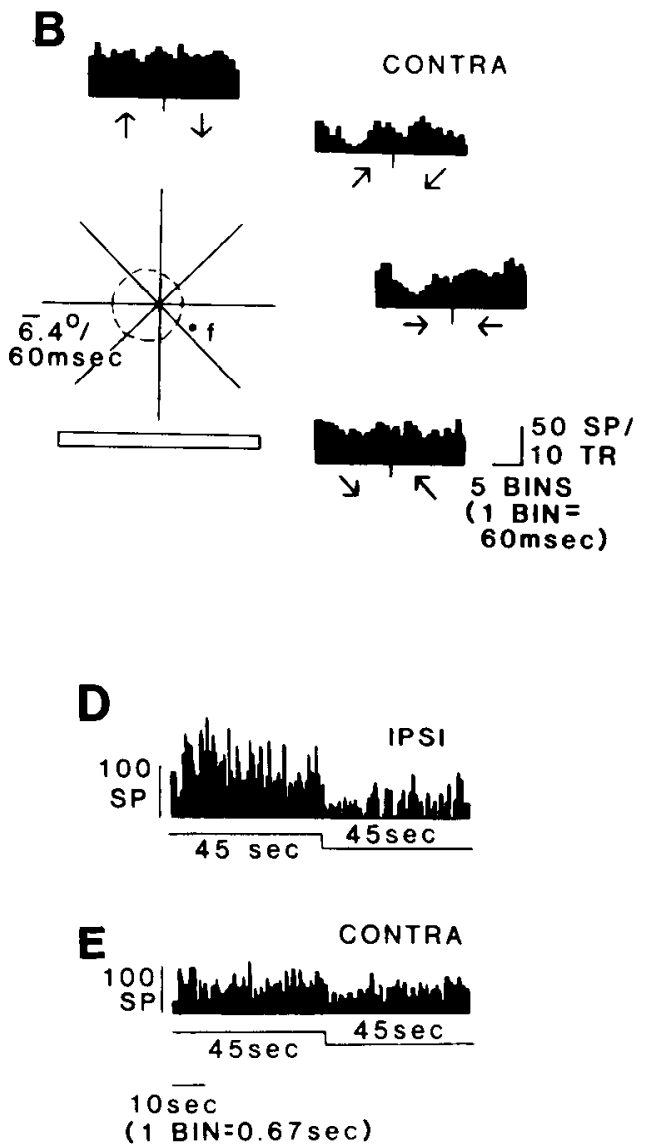

Figure 5. Sustained responses tu steady illumination-excitation. $A$ to $C$ illustrate the response of a $\mathrm{PL}_{\gamma}$ unit to a moving bar of light $\left(69.3^{\circ} \times 5.7^{\circ}\right.$ in size; velocity $\left.=106.7^{\circ} / \mathrm{sec}\right)$ under binocular (both) and monocular conditions. In each case, a unidirectional inhibition is the dominant response, especially at the $0^{\circ}$ and $45^{\circ}$ trajectories. During steady state illumination of the ipsilateral eye $(D)$, however, the unit exhibits a pronounced excitation which returns to spontaneous levels during the dark. The contralateral eye $(E)$ seems unaffected by luminance changes. The response to steady state illumination in the binocular condition is similar to that in the ipsilateral condition. See Figure 1 for the location of this unit.

contracting disks and since the majority of these units could respond to approaching or receding stimuli in the monocular condition, it appears that their ability to respond to motion in depth is a function of the changing size of stimuli rather than a stereoscopic mechanism. That is, the demonstrated ability of these units to respond to motion in depth is not due to parallax between the two eyes but to the changing size of the retinal image.

The unit in Figure 6 is one in which the bilateral receptive fields were $55^{\circ}$ in diameter and included the fovea. It was clear that the response to a moving bar of light was similar for both eyes, but it was not possible to determine any sensitivity to stationary flashes. The unit was strongly driven when a back-lighted black disk, centered on the receptive field, was moved away from or toward the animal's eyes by a transducer. With this type of stimulation, the unit responded most strongly during the binocular condition, although it was noticeably affected by stimulation of the ipsilateral eye alone. It was not noticeably influenced by monocular stimulation of the contralateral eye. At all times, the disk was contained within the boundaries of the receptive field. The rate of change of the disk's apparent size in the animal's receptive field is given by the following equation which is derived in the legend to Figure 6:

$$
\begin{aligned}
\theta(t)=\frac{d}{d t}\left[2 \tan ^{-1} \frac{r}{K t+D_{0}}\right] & \\
& =\frac{360 r K}{\pi\left(K^{2} t^{2}+D_{0} K t+D_{0}^{2}+r^{2}\right)} \text { in degrees/sec }
\end{aligned}
$$

where $r$ is the radius of the disk, $K$ is the rate of movement, and $D_{0}$ is the initial distance of the disk from the animal. As shown by Figure $6 C, D$, the rate of discharge of this unit, is, in fact, proportional to this function. Note that a discontinuity exists in the derivative at about 1 sec due to the use of a triangular waveform to drive the movement of the disk (Fig. 6).

It should be pointed out that, with these units, it was not possible to tell from the responses to tangentially moving or stationary stimuli whether the unit would be sensitive to movement toward or away from the eyes. These units behaved in the same way to tangentially moving stimuli as those units which had bilateral or contralateral receptive fields but were not sensitive to stimuli which moved away from or toward the eyes (see "Discussion").

Non-visual stimuli. The $\mathrm{PL}_{\gamma}$ units which responded to visual stimuli also were generally tested with auditory and somatosensory stimuli.

The units encountered in this study seemed most 

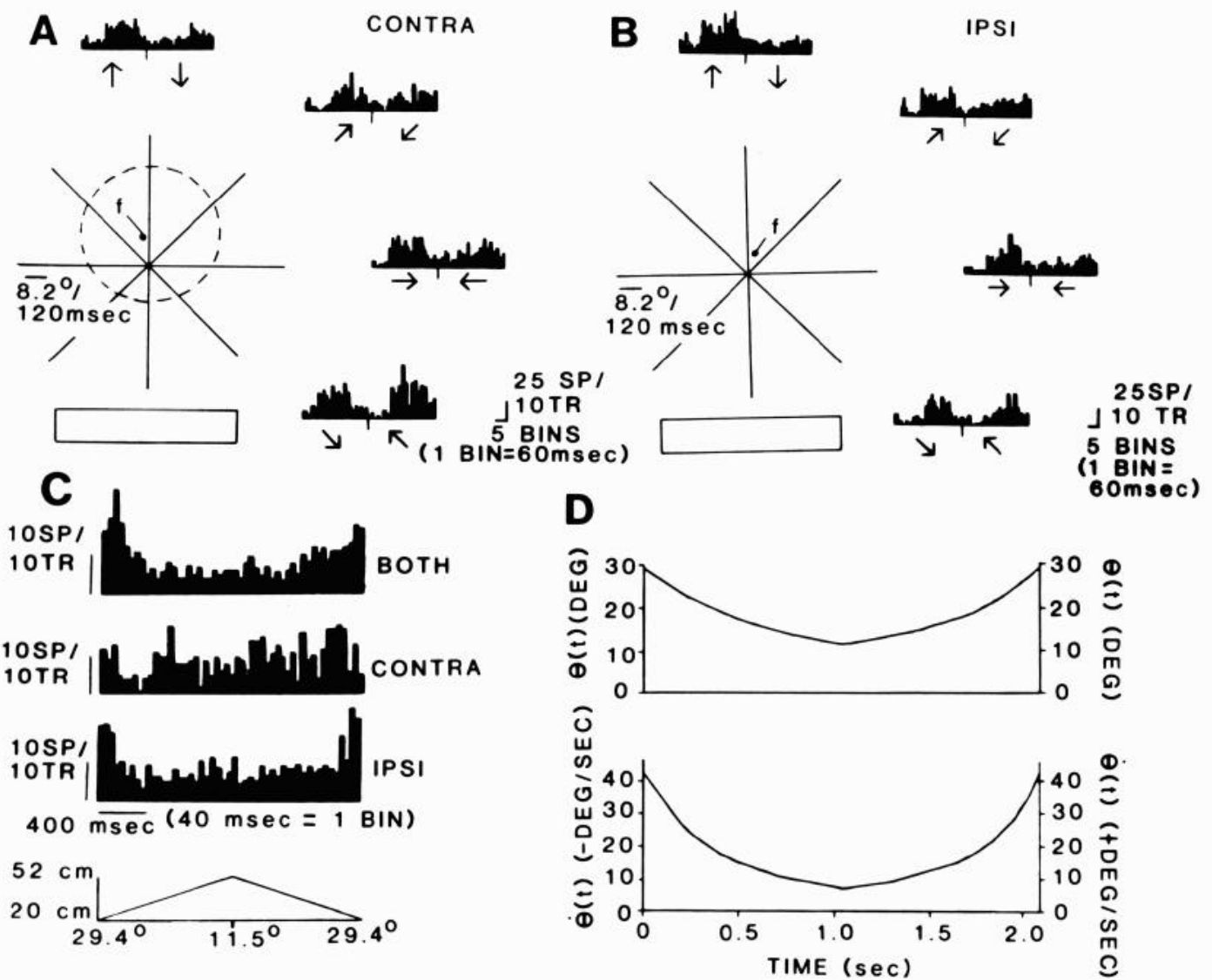

Figure 6. Responses to movement in depth. Panels $A$ and $B$ illustrate the monocular responses of a unit in $\mathrm{PL}_{\gamma}$ to a light bar $67.1^{\circ} \times 12.7^{\circ}$ in size moving at $68.3^{\circ} / \mathrm{sec}$. The unit is clearly excited by the stimulus and may be directionally selective. Panel $C$ illustrates the response of this unit to a black disk which is moved toward and then away from the eyes. Both monocular and binocular conditions are shown. A response is elicited from the ipsilateral eye alone, but it is difficult to determine if the contralateral eye is also sensitive. The clearest response comes from the binocular condition. In this particular case, the stimulus consists of a dark disk, $10.5 \mathrm{~cm}$ in diameter, which is moved from a near distance of $20 \mathrm{~cm}$ to a far distance of $52 \mathrm{~cm}$ at a linear speed as shown at the bottom of panel $C$. The histograms in panel $C$ show that unit's response to this stimulus condition. Given a stimulus that moves radially to the experimental animal at a linear rate of speed, it is possible to derive $\theta(t)$, the angle of visual field taken up by the stimulus as a function of time, and $\theta^{\prime}(t)$ is the rate of change of this angle. $\theta(D)=2 \tan ^{-1}(R / D)$, where $D$ is distance and $R$ is the radius of the disk. If $D(t)$ is expressed as $K t+D_{0}$, where $K$ is a constant and $D_{0}$ is the distance from the eyes at $t=0$, then $\theta(\mathrm{t})=2 \tan ^{-1}\left(R / K t+D_{0}\right)$. This function is drawn at the top of panel $D$. This function can be differentiated, giving $\theta^{\prime}(t)$, which is equal to $2 R K /\left(K^{2} t^{2}+2 D_{0} K t+D_{0}^{2}+R^{2}\right)$ in radians per sec or $360 \cdot R K / \pi\left(K^{2} t^{2}+2 D_{0} K t+D_{0}{ }^{2}+R^{2}\right)$ in degrees per second. This function is shown at the bottom of panel $D$. Note especially the resemblance between this function and the histogram at the top of panel $C$. While this function holds at other velocities, the unit's sensitivity to movement in depth appears to be restricted to a very narrow range of velocities. See Figure 1 for the location of this unit.

clearly influenced by visual stimuli whether moving or not. Non-visual stimuli usually had no noticeable effect except for one or two cells which might have reacted weakly to somatosensory stimulation.

\section{Discussion}

The results of this investigation report, for the first time, the visual properties of units in that portion of the pulvinar complex $\left(\mathrm{PL}_{\gamma}\right)$ which provides a major afferent input to the visual association cortex in the macaque monkey. These results can be summarized as follows: (1) the anatomically defined caudal subdivision of the lateral pulvinar $\left(\mathrm{PL}_{\gamma}\right)$ has a poor and disorganized retinotopic organization; (2) the neurons have large, unflanked (often bilateral) receptive fields and a disproportionate amount of units have receptive fields which are located in the region of central vision; (3) the majority of units are binocular and can exhibit complex types of interactions; (4) most neurons are sensitive to moving stimuli; (5) some units which are sensitive to tangentially moving stimuli also give sustained responses to static levels of luminance; (6) some units which respond to tangentially moving stimuli also respond to stimuli which move toward or away from the eyes; and (7) for many of these luminance-sensitive units, binocular units, and units sensitive to moving stimuli, the responses to one class of stimuli are seemingly unrelated to the responses to another class of stimuli.

Organization of $P L_{r}$. We did not find a clear systematic map of the contralateral visual hemifield in $\mathrm{PL}_{\gamma}$. The receptive fields overlap a great deal; many are large and about half are bilateral. Thus, the presence of any systematic map may have been obscured by this type of organization. If one considers that the large receptive 
fields (bilateral or unilateral) can represent the sum of smaller receptive fields located in either hemifield, then any systematic map of the contralateral hemifield would have been lost as its receptive fields became integrated into the larger overlapping receptive fields. However, we also must consider the possibility that our penetrations traversed one or more systematic maps in such a way as to produce a distorted picture.

The lack of a clear retinotopic organization, the presence of bilateral receptive fields, and a predominance of receptive fields which involve the fovea should not be too surprising in light of recent anatomical reports on the connections of $\mathrm{PL}_{\gamma}$ (e.g., Benevento and Davis, 1977; Rezak, 1978). While regions of the lateral pulvinar receive inputs from the midbrain (Benevento and Fallon, 1975; Benevento et al., 1977b; Harting et al., 1980; Standage and Benevento, 1981; L. A. Benevento and G. Standage, submitted for publication), a major source of input to the entire lateral pulvinar is from the cortex (Benevento and Davis, 1977; Rezak, 1978). It has been shown that, while dorsal prestriate ${ }^{3}$ areas seem to project to dorsal $\mathbf{P L}_{\gamma}$ and ventral prestriate areas project to ventral $\mathrm{PL}_{\gamma}$, any further topography to the organization of cortical inputs was difficult to determine (Benevento and Davis, 1977). Rather, the projection fields in $\mathrm{PL}_{\gamma}$ from any given, small region of prestriate cortex consist of several elongated foci or bands which overlapped with similar projection foci arising from other focal and widely separated regions in prestriate cortex. This is in contrast to more rostral regions of the pulvinar complex where both the ventral portion of the rostral lateral pulvinar $\left(\mathrm{PI}_{\alpha_{\alpha}}\right)$ and adjacent inferior pulvinar have retinotopically organized reciprocal connections with the visual cortex, e.g., the striate cortex. The convergent cortical input to $\mathrm{PL}_{\gamma}$ can explain why many of the receptive fields are large and bilateral. It would also explain why there is a "jumping around" of receptive field positions from peripheral to more central and vice versa as one records from adjacent points in the nucleus (Fig. 1). It has been argued before that this convergent prestriate input to $\mathrm{PL}_{\gamma}$ may be important in terms of the strong projection from $\mathrm{PL}_{\gamma}$ to the visually responsive inferotemporal cortex (areas 20 and 21) (Benevento and Rezak, 1976; Rezak, 1978). It is interesting to note that, like $\mathrm{PL}_{\gamma}$, the inferotemporal cortex is not retinotopically organized and has many bilateral receptive fields that include the fovea. The range of receptive field sizes is similar for both $\mathrm{PL}_{\gamma}$ and the inferotemporal cortex and includes a group of receptive fields which are large and whose borders cannot be detected reliably (Gross et al., 1972; Desimone and Gross, 1976). Prestriate cortex, on the other hand, has retinotopically organized regions and smaller receptive fields which usually are confined to the contralateral visual field. Moreover, in both $\mathrm{PL}_{\gamma}$ and the inferotemporal cortex, units exhibit binocularity (with varying degrees of eye dominance), a preference for moving stimuli, directional selectivity, and monocular and binocular sen-

\footnotetext{
${ }^{3}$ Prestriate cortex is defined as the visual association cortices of the occipital lobe, i.e., areas 18 and 19. This region is also known as "extrastriate" or "peristriate" cortex and consists of a number of functional areas (Zeki, 1978).
}

sitivity to three-dimensional objects. As a result of the present study, there seems to be no good reason to modify our previous notions of the organization of the projections of $\mathrm{PL}_{\gamma}$ to the inferotemporal cortex. That is, the occipital cortex can influence the inferotemporal cortex via its convergent inputs to $\mathrm{PL}_{\gamma}$, thus forming a corticothalamocortical circuit.

However, we must not be too quick to ignore sources of input to the lateral pulvinar which arise from regions other than the cortex. Anatomical studies (Benevento and Fallon, 1975; Benevento et al., 1977b; Harting et al., 1980; Magnuson et al., 1979; Standage and Benevento, 1980, 1981; L. A. Benevento and G. Standage, submitted for publication) have shown that the lateral pulvinar (and adjacent regions of the medial pulvinar) contain segregated islands of inputs which are from the pretectum and superior colliculus. The fact that units with peripheral receptive fields and sensitivity to the orientation of a tangentially moving stimulus tend to be found in dorsomedial $\mathrm{PL}_{\gamma}$ (which is adjacent to the medial pulvinar) can be explained by selective midbrain inputs to this particular region (Benevento and Fallon, 1975; Benevento et al., 1977b; Standage and Benevento, 1981; Wurtz and Albano, 1980; I. A. Benevento and G. Standage, submitted for publication). It would not be surprising if other variations in response properties throughout an apparently homogeneous region of the pulvinar can be explained by these regional variations in midbrain inputs (Standage and Benevento, 1981; L. A. Benevento and G. Standage, submitted for publication). In fact, in one of our more recent studies, we have found several latency classes in the lateral pulvinar which correlate well with different response properties including color sensitivity (e.g., Felsten et al., 1981).

Bilateral receptive fields are more difficult to explain. The large bilateral receptive fields overlap the smaller receptive fields in $\mathrm{PL}_{\gamma}$ which are located in either hemifield and, thus, could represent a local convergence of pulvinar cells with smaller receptive fields located in either hemifield. This would help account for a lack of a clear systematic map of the contralateral hemifield as mentioned in the beginning of the discussion (p. 1275). Another source of bilateral organization can be from feedback from the occipital or inferotemporal cortex to the pulvinar. The bilateral receptive fields found in the cortex are created by interhemispheric convergence via the corpus callosum (Rocha-Miranda et al., 1975). In addition, portions of the pretectum or superior colliculus which receive ipsilateral and contralateral inputs from the retina can have overlapping convergent inputs to the lateral pulvinar (Benevento and Fallon, 1975; Benevento and Rezak, 1977; Benevento et al., 1977b; Felsten et al., 1981; Magnuson et al., 1979; Standage and Benevento, 1980, 1981; L. A. Benevento and G. Standage, submitted for publication).

Static and dynamic responses. Unit sensitivity to both moving and stationary stimuli was demonstrated in this study (e.g., Figs. 4 and 5). Apparently the same unit could respond with excitation or inhibition to a moving bar of light and give the exact opposite response to stationary, diffuse whole field illumination. Two points can be made. The first concerns the characteristics of these sustained 
responses during steady levels of luminance alone and the second concerns the apparent independence of the static and dynamic responses.

Units which alter their firing rates during prolonged, steady periods of light or dark have been described before in primary visual cortex (e.g., Bartlett and Doty, 1974; Kayama et al., 1979) and have been called "luxotonic." Most of these units are binocular and some of their receptive fields actually cross the vertical meridian. The rostral lateral pulvinar projects to the primary visual cortex (Rezak and Benevento, 1979) and this may be the substrate by which the presence of bilateral, binocular luxotonic units in striate can be explained. The geniculate also has been shown to contain luxotonic units (Kayama et al., 1979), but, unlike the cortex and the pulvinar, other properties, such as bilateral organization, are not seen with geniculate cells, although the geniculate has been shown recently to project to the visual association cortex in the macaque monkey (Benevento and Yoshida, 1981; Yoshida and Benevento, 1981). Inputs to the inferotemporal cortex from the lateral pulvinar which signal sustained levels of brightness or darkness would be useful during visual pattern discrimination which is a function for which the inferotemporal cortex is the substrate.

As seen in the present study, it appears that the responses of pulvinar units to static and dynamic stimuli are not predictable from each other. This organization in which opposite responses are obtained from luminance levels and moving stimuli seems to be achieved by different synaptic pathways converging upon the same cell. This concept is supported by differences in the apparent receptive fields' organizations of these units as defined by stationary and moving stimuli. While many of these cells appeared to have a discrete region of sensitivity or a restricted receptive field as defined by moving stimuli, luminance changes anywhere within the entire visual field produced sustained responses. These different synaptic pathways might be useful in pattern discrimination by "increasing the contrast" (i.e., by exaggerating the difference (to the unit) between the background illumination and transiently occurring stimuli and their salient features).

Movement toward or away. An interesting group of units responded to stimuli which approached or retreated from the eyes (Fig. 6). These units were sensitive to such stimuli under both monocular and binocular conditions and the key cue seems to be the change in size of the retinal image. These cells also responded to single, tangentially moving stimuli. Units sensitive to movement toward or away from the animal have been described in striate and prestriate cortex (Talbot and Poggio, 1978; Zeki, 1974). Apparently a number of these units, such as those in foveal striate cortex, are not obviously sensitive to depth unless they are actually tested with stimuli that move in depth (Talbot and Poggio, 1978). Other depthsensitive neurons may be predicted by their sensitivity to fixed disparily lesting (Talbot and Poggio, 1978) or to opposed movement of two edges within the receptive field (Zeki, 1974). In the latter experiment, the cells were insensitive to movement of a single edge. In contrast to this latter experiment, we used stimuli which actually moved in depth and the same pulvinar cells which were sensitive to movement in depth were also sensitive to single tangentially moving edges and bars. It again seems that responses to one set of stimuli do not necessarily predict the responses to another set.

The existence of such units in $\mathrm{PL}_{\gamma}$ indicates that this system is involved in more than visual discrimination but is important in visuomotor function, such as movement in three-dimensional space as the animal explores the world.

Comparison to New World monkeys. The other full reports on the response properties of pulvinar neurons come from work using New World monkeys.

We did not find obviously flanked receptive fields in this study in the macaque monkey. Thus, our units resemble the static neurons with diffuse, uniform rcceptive fields and the dynamic movement-sensitive units reported for the lateral pulvinar of the squirrel monkey (Mathers and Rapisardi, 1973). Unlike the neurons in the squirrel monkey lateral pulvinar, however, we were unable to demonstrate sensitivity to somatosensory stimuli.

The reason that we did not detect any sensitivity to somatosensory stimuli may be due to the location of the recording sites in the pulvinars of the squirrel monkey and macaque monkey. We would expect cells in the more rostral and dorsal portions of the lateral pulvinar of the macaque to respond to somatosensory stimuli as this region, termed $\mathrm{PL}_{\beta}$ by Rezak and Benevento (1977), has connections with the parietal cortex (Benevento et al., 1977b; Rezak and Benevento, 1979; Motter and Mountcastle, 1981).

Some of the responses of the macaque pulvinar neurons also resemble some reported for the cebus monkey (Gattas et al., 1979). These would be their static and dynamic uniform receptive field types (i.e., groups 1, 4, and 6). The dynamic units in that study exhibited a sensitivity to direction of movement and binocular interaction and also could have bilateral receptive fields. The majority of our units, like group 6 in the cebus monkey, had for orientation selectivity a "great tolerance for stimulus deviation." The degree of involvement of the ipsilateral hemifield was much less than seen in the present study and no receptive fields were entirely within the ipsilateral hemifield as we have found. The region of the cebus pulvinar which was studied probably does not correspond to $\mathrm{PL}_{\gamma}$ but corresponds more closely to both the inferior pulvinar and region $\mathrm{PL}_{\alpha}$ of the macaque (Rezak and Benevento, 1977, 1979; Benevento and Davis, 1977). Our results in the macaque do not correspond to those in the cebus monkey where neurons which exhibited flanked receptive fields as well as orientation sensitivity were common. These and other discrepancies may be due not only to different recording sites within the pulvinar complex but also to species differences.

\section{References}

Bartlett, J. R., and R. W. Doty (1974) Response of units in striate cortex of squirrel monkeys to visual and electrical stimuli. J. Neurophysiol. 37: 621-641.

Benevento, L. A., and P. D. Coleman (1970) Responses of single cells in cat inferior colliculus to binaural click stimuli: Combination of intensity levels, time and intensity differences. Brain Res. 17: 387-405.

Benevento, L. A., and P. Davis (1977) Topographical projec- 
tions of the prestriate cortex to the pulvinar nuclei in the macaque monkey: An autoradiographic study. Exp. Brain Res. 30: 405-424.

Benevento, L. A., and J. H. Fallon (1975) The ascending projections of the superior colliculus in the rhesus monkey (Macaca mulatta). J. Comp. Neurol. 160: 339-362.

Benevento, L. A., and M. Rezak (1976) The cortical projections of the inferior pulvinar and adjacent lateral pulvinar in the rhesus monkey (Macaca mulatta): An autoradiographic study. Brain Res. 108: 1-24.

Benevento, L. A., and M. Rezak (1977) Further observations on the projections of the layers of the superior colliculus in the rhesus monkey with autoradiographic tracing methods. Soc. Neurosci. Abstr. 3: 553

Benevento, L. A., and K. Yoshida (1981) The afferent and efferent organization of the lateral geniculo-prestriate pathways in the macaque monkey. J. Comp. Neurol. 202: 203.

Benevento, L. A., O. D. Creutzfeldt, and U. Kuhnt (1972) Significance of intracortical inhibition in visual cortex. $\mathrm{Na}$ ture New Biol. 238: 124-126.

Benevento, L. A., J. H. Fallon, P. Davis, and M. Rezak (1977a) Auditory-visual interaction in single cells in the cortex of the superior temporal sulcus and the orbital frontal cortex of the macaque monkey. Exp. Neurol. 57: 849-872.

Benevento, L. A., M. Rezak, and R. Santo-Anderson (1977b) An autoradiographic study of the projections of the pretectum in the rhesus monkey (Macaca mulatta): Evidence of sensorimotor links to the thalamus and oculomotor nuclei. Brain Res. 127: 197-218.

Chow, K. L. (1954) Effects of temporal neocortical ablation on visual discrimination learning sets in monkeys. J. Comp. Neurol. 47: 194-198.

Desimone, R., and C. Gross (1976) Absence of retinotopic organization in inferior temporal cortex. Soc. Neurosci. Abstr. 2: 1108.

Ettinger, G., E. Iwai, M. Mishkin, and H. E. Rosvold (1968) Visual discrimination in the monkey following serial ablation of inferotemporal and preoccipital cortex. J. Comp. Physiol. Psychol. 65: 110-117.

Fallon, J., and L. A. Benevento (1977) Auditory-visual interaction in cat orbital-insular cortex. Neurosci. Lett. $6: 143-149$.

Fallon, J., L. A. Benevento, and P. R. Loe (1978) Frequencydependent inhibition to tones in neurons of insular cortex (AIV). Brain Res. 144: 149-154.

Felsten, G., D. Burman, K. Yoshida, and L. A. Benevento (1981) Visual response properties of single neurons in the macaque monkey pulvinar. Soc. Neurosci. Abstr. 7: 759.

Gattas, R., E. Oswaldo-Cruz, and A. P. B. Sousa (1979) Visual receptive fields of units in the pulvinar of cebus monkey. Brain Res. 160: 413-430.

Gross, C. G., C. E. Rocha-Miranda, and D. B. Bender (1972) Visual properties of neurons in inferotemporal cortex of the macaque. J. Neurophysiol. 35: 96-111.

Harting, J., M. Huenta, A. S. Frankfurter, N. Strominger, and G. L. Royce (1980) Ascending pathways from the monkey superior colliculus: An autoradiographic study. J. Comp. Neurol. 192: 852-882.

Iwai, E., and M. Mishkin (1969) Further evidence on the locus of the visual area in the temporal lobe of the monkey. Exp. Neurol. 25: 585-594.

Kayama, Y., R. R. Riso, J. R. Bartlett, and R. W. Doty (1979) Luxotonic response of units in macaque striate cortex. J. Neurophysiol. 42: 1495-1517.

Magnuson, D., M. Rezak, and L. A. Benevento (1979) Some observations on the organization of the retinal projections to the pretectum and superior colliculus in the macaque monkey as demonstrated by the combined use of laser beam lesions of the retina and autoradiography. Soc. Neurosci. Abstr. 5: 794.

Mathers, L. H., and S. C. Rapisardi (1973) Visual and somatosensory receptive fields of neurons in the pulvinar of the squirrel monkey. Brain Res. 64: 65-84.

Motter, B. C., and V. B. Mountcastle (1981) The functional properties of the light-sensitive neurons of the posterior parietal cortex studied in waking monkeys: Foveal sparing and opponent vector organization. J. Neurosci, 1: 3-26.

Rezak, M. (1978) Some observations on the organization of the caudal pole of the thalamus (lateral pulvinar or $\mathrm{PL}_{\gamma}$ ) in the macaque monkey. Soc. Neurosci. Abstr. 4: 642.

Rezak, M., and L. A. Benevento (1977) A redefinition of pulvinar subdivisions in the macaque monkey: Evidence for three distinct subregions within classically defined lateral pulvinar. Soc. Neurosci. Abstr. 3: 573.

Rezak, M., and L. A. Benevento (1979) A comparison of the projections of the dorsal lateral geniculate nucleus, the inferior pulvinar and adjacent lateral pulvinar to striate cortex (area 17) in the macaque monkey. Brain Res. 167: 19-41.

Rocha-Miranda, C. E., D. B. Bender, C. G. Gross, and M. Mishkin (1975) Visual activation of neurons in inferotemporal cortex depends upon striate cortex and forebrain commissures. J. Neurophysiol. 38: 475-491.

Standage, G., and L. A. Benevento (1980) Relationship of subdivisions of the pulvinar complex to the various visual areas within the occipital lobe of macaque monkeys. Soc. Neurosci Abstr. 6: 481.

Standage, G., and L. A. Benevento (1981) Multiple midbrain inputs to the lateral pulvinar with reference to certain of their cortical projections. Soc. Neurosci. Abstr. 7: 830.

Talbot, W. H., and G. F. Poggio (1978) Activity of neurons in visual cortex of the alert macaque evoked by stationary and moving stimuli in three dimensional space. Soc. Neurosci. Abstr. 4: 648.

Walker, A. E. (1938) The Primate Thalamus, University of Chicago Press, Chicago.

Wurtz, R. H., and J. E. Albano (1980) Visual-motor function of the primate superior colliculus. Annu. Rev. Neurosci. 3: 189226.

Yoshida, K., and L. A. Benevento (1981) The projection from the dorsal lateral geniculate of the thalamus to extrastriate visual association cortex in the macaque monkey. Neurosci. Lett. 22: 103-108.

Zeki, S. M. (1974) Cells responding to changing image size and disparity in the cortex of the rhesus monkey. J. Physiol. (Lond.) 242: 827-841.

Zeki, S. (1978) Functional specialization in the visual cortex of the rhesus monkey. Nature 274: 423-429. 